\title{
The Companion CD-ROM
}

The companion CD-ROM for Stars as Suns: Activity, Evolution, and Planets includes all of the papers in this printed volume (IAU Symposium 219) and the poster papers that were submitted for publication. The poster papers (denoted in the Table of Contents by the pagination CD-xxx) can only be found in the CDROM. These poster papers are individually paginated and should be referenced in a similar way as the papers in the book, but with a page reference prefaced by "CD-" as it appears on the pages of the papers.

The top level directory contains a file called index.html which is the hypertext 'home page' for the CD-ROM and has links to the Table of Contents, and the Author Index.

The papers themselves are denoted by the first author's last name, followed by either a $p d f$ extension or a $p s . g z$ extension. In the case of multiple papers by the first author, they are sequenced with a running number starting with " 1 " following the authors name.

Scientific papers presented in $p d f$ format can be read with an Adobe PDF Document Viewer such as Adobe Acrobat and/or Acroread. The program Adobe Reader is available as free software for viewing and printing Adobe Portable Document Format (PDF) files. It can be downloaded for many platforms from the website: http://www.adobe.com/products/acrobat/readstep2.html

Because many papers contain detailed graphical material, a pdf file does not reproduce them well. Therefore these papers are stored as compressed Postscript files. They can be uncompressed by using the gunzip utility, and then read with any Postscript viewer, such as ghostview.

Color and black and white photographs from the meeting, taken by and kindly provided by Scott Wolk are included in jpg format. Many of these appear also in the book. The conference poster is also included.

This CD-ROM has been tested in computers using UNIX/Linux and Windows (Microsoft) platforms; please consult the documentation for your particular setup.

We are grateful to the IAU for supporting the production of this CD-ROM and its inclusion in the Symposium volume. 


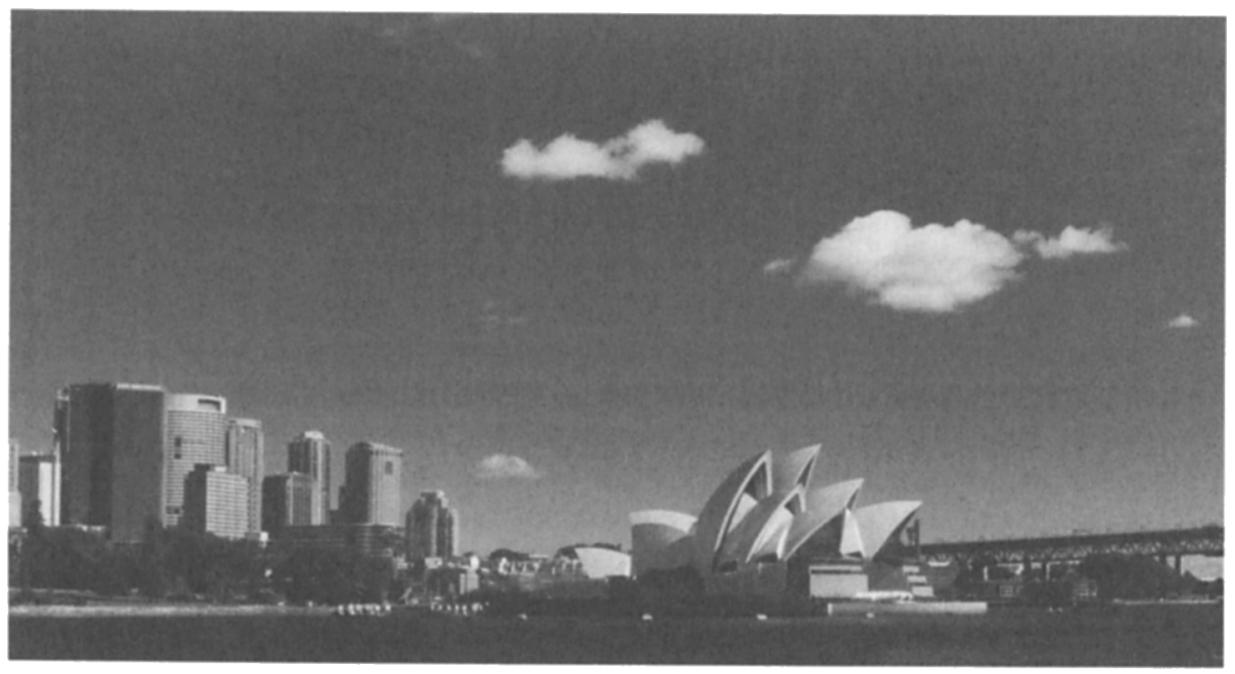

